Research Paper

\title{
Immunohistochemical Evaluation of Neuroreceptors in Healthy and Pathological Temporo-Mandibular Joint
}

Gianfranco Favia ${ }^{1}$, Massimo Corsalini ${ }^{\circledR}{ }^{\bowtie}$, Daniela Di Venere ${ }^{2}$, Francesco Pettini² ${ }^{2}$ Giorgio Favia ${ }^{3}$, Saverio Capodiferro4, Eugenio Maiorano ${ }^{5}$

1. Full Professor, Dental School, University of Bari, Bari, Italy

2. Assistant Professor, Dental School, University of Bari, Bari, Italy

3. Postgraduate in Plastic surgery, Campus Biomedico, University of Rome, Rome, Italy

4. Lecturer, Dental School, University of Bari, Bari, Italy

5. Full Professor, Department of Pathological Anatomy, University of Bari, Bari, Italy

$\triangle$ Corresponding author: Massimo Corsalini. Dental School, University of Bari, Bari, Italy. Piazza Giulio Cesare, 10. CAP 70121 Bari, Italy. Tel: +39805478153. Fax: +39805478743. E-mail: massimo.corsalini@uniba.it

(C) Ivyspring International Publisher. This is an open-access article distributed under the terms of the Creative Commons License (http://creativecommons.org/ licenses/by-nc-nd/3.0/). Reproduction is permitted for personal, noncommercial use, provided that the article is in whole, unmodified, and properly cited.

Received: 2013.03.24; Accepted: 2013.08.13; Published: 2013.09.25

\begin{abstract}
Aim: A study was performed on the articular disk and periarticular tissues of the temporo-mandibular joint (TMJ) with immunohistochemical techniques to give evidence to the presence of neuroreceptors (NRec) in these sites. Methods: The study was carried out on tissue samples obtained from 10 subjects without TMJ disease and from 7 patients with severe TMJ arthritis and arthrosis. We use antibodies directed against following antigens: Gliofibrillary Acidic Protein (GFAP), Leu-7, Myelin Basic Protein (MBP), Neurofilaments 68 kD (NF), Neuron Specific Enolase (NSE), S-100 protein (S-100) and Synaptophysin (SYN). Results: This study revealed that Ruffini's-like, Pacini's-like and Golgi's-like receptors can be demonstrated in TMJ periarticular tissues and that free nervous endings are present in the subsynovial tissues but not within the articular disk. We observed elongated cytoplamic processes of chondrocytes that demonstrated strong S-100 immunoreactivity but they were unreactive with all other antibodies. These cytoplamic processes were more abundant and thicker in the samples obtained from patients with disease TMJ. Conclusion: The results of this study confirm that different $\mathrm{Nrec}$ are detectable in TMJ periarticular tissues but they are absent within the articular disk. In the latter site, only condrocytic processes are evident, especially in diseased TMJ, and they might have been confused with nervous endings in previous morphological studies. Nevertheless the absence of immunoreactivity for NF, NSE and SYN proves that they are not of neural origin.
\end{abstract}

Key words: neuroreceptors, temporo-mandibular joint

\section{Introduction}

The function of sensory receptors (Nrec) in the movement control, muscle coordination and perception of the space position of temporo-mandibular joint (TMJ) is fundamental although the presence of Nrec in the TMJ is still debated: some authors have reported on the lack of nervous fibers in the articular disk $(1,2)$, while florid innervation of TMJ has been reported in several studies on animal models and in human (3-6) which suggested that (6) the concentra- tion of sensory receptors within TMJ is higher in the areas supporting higher strong tensions during articular movements (chewing, biting, speaking). In discordance, other authors disclosed the presence of mechanical Nrec in the articular disk of human TMJ $(7,8)$ also distinguishing receptors in capsulated and uncapsulated on the bases of morphological features.

The aim of this study is to ascertain the presence and the distribution of Nrec in human TMJ by using 
of immunohistochemical investigations in healthy and pathological TMJ such as arthritis and arthrosis. The study was approved by the Bioethics Committee of the Department of Odontology and Surgery of University of Bari.

\section{Materials and Methods}

Totally 17 cases were studied. 10 samples of capsular and pericapsular soft tissues with the disk were obtained from healthy patients (six men and four women with a mean age of 39 years) who suffered surgery of TMJ because of accidental trauma of the temporo-mandibular region; the remaining 7 cases (four men and three women with a mean age of 57 years) were patients surgically treated for severe degenerative lesions of TMJ (chronic arthritis and arthrosis). All specimens were immediately fixed in neutral buffered formalin and embedded in paraffin; 5 micron thick sections were cut and stained with Haematoxylin-Eosin, PAS, Gomori's reticulin and Azan-Mallory trichrome; consecutive sections were used for the immunohistochemical detection of the antigens listed in table 1 . All the antibodies used are commercially avalaible from Dako Italia Spa, Milan, Italy (Glial Fibrillary Acidic Protein = GFAP; Myelin Basic Protein = MBP; Neurofilaments = NF; Neuron Specific Enolase = NSE; Synaptophysin; S-100 Protein = S-100) and from Becton Dickinson, Burlingame, USA (Leu-7). In all cases the immunohistochemical Alkaline phosphatase-anti-alkaline phosphatase (APAAP) method was performed (9) while some sections for anti-Neurofilaments antibodies were treated for 10 minutes with $1 \%$ Saponin in Phosphate Buffered Saline (PBS), Ph 7.2, before the application of primary antibodies.

Table I. List of the antibodies used to immunocharacterize sensory neuroreceptors in TMJ.

\begin{tabular}{|c|c|c|}
\hline Antigen detected & Dilution & Reactivity \\
\hline GFAP & $1: 100$ & central and peripheral glial cells \\
\hline Leu-7 & 1:10 & $\begin{array}{l}\text { CD 57, glycoprotein of myelinic } \\
\text { envelopes }\end{array}$ \\
\hline MBP & $1: 50$ & protein of the myelinic envelope \\
\hline $\begin{array}{l}\text { Neurofilaments } \\
68 \mathrm{KD}\end{array}$ & 1:100 & $\begin{array}{l}\text { neural cells and their dendrites and } \\
\text { axons }\end{array}$ \\
\hline NSE & $1: 30$ & $\begin{array}{l}\text { neural cells and their dendrites and } \\
\text { axons }\end{array}$ \\
\hline S-100 protein & $1: 300$ & $\begin{array}{l}\text { neural and Schwann cells, melano- } \\
\text { cytes, myoepithelial cells, chondro- } \\
\text { cytes, lipocytes }\end{array}$ \\
\hline Synaptophysin & $1: 50$ & protein of synaptic vesicles \\
\hline
\end{tabular}

\section{Results}

With histochemical techniques and H\&E stain, Nrec were not easily detectable (Figures 1-8); howev- er, these were used to identify the corresponding tissue in immunostained slides.

In all specimens, such types of Nrec have been detected by immmunohistochemistry (table 2):

a) globular receptors with a thin capsule closely resembling Ruffini's ones; they exhibited strong immunoreactivity in their core for S-100, NSE and Leu-7 and were abundant in the superficial peri-articular muscles and in the peri-articular fibrous capsule; $b$ ) elongated, onion-like receptors with a thick capsule mimicking Pacini's receptors, positive for MBP, S-100, NSE and Leu-7, particularly abundant in deep muscle fibres and in peri-articular dense fibrous tissues; c) fusiform capsulated receptors, morphologically similar to Golgi's receptors, located within peri-articular fibrous tissues and ligament and fibromuscolar resections, strongly positive for S-100, MBP and Leu-7 and weaker GFAP reactivity.

In all these types of Nrec $(a+b+c)$ and especially in the core, punctate reactivity for Synaptophysin and for Neurofilaments was also evident; d) free and thin nervous endings in high density within subsynovial connective tissues, in intra- and periarticular fibrous tissues and along the perimisial, endomisial sarcolemma of striated muscle fibres were detected, showing immunoreactive for Neurofilaments, NSE, Synaptophysin and to S-100 antibodies.

Table 2. Distribution of neural receptors in TMJ tissues

Extra pericapsular location (mainly)
- Type 1 (Ruffini-like): superficial layers
- Type 2 (Pacini-like): deeper layers
- Type 3 (Golgi-like): pericapsular fibrous tissues
Intracapsular locations (mainly)
- Type 4 (non capsulated): free endings with diffuse intra and ex-
tra-articular perivascular distribution with high density in intra-
muscolar and in post-discal soft tissues.
- Elongated cytoplamic processes of chondrocytes demonstrated
strong S-100 immunoreactivity but they were unreactive with all
other antibodies. These cytoplamic processes were more abundant
and thicker in the samples obtained from patients with diseased
TMJ.

In the articular fibrous cartilage (articular disk) any previously described Nrec was identified; only S-100 protein seemed to react with chondrocytes both in normal and diseased tissues. Furthermore, chondrocytes of healthy individuals appeared round-shaped, with distinct cell borders and central nuclei with an evident S-100 reactivity both in nucleus and in cytoplasm. In diseased TMJ, instead, chondrocytes showed a different morphoology, especially after S-100 immunostaining: they had an elongated cytoplasm with one or more thick dendritiform processes of variable length but with a strong reactivity 
only for S-100 protein. The number of dendritiform chondrocytes was higher in specimens of diseased patients than in healthy patients and seems undergo a reactive reparative proliferation of discal and peridiscal tissues.

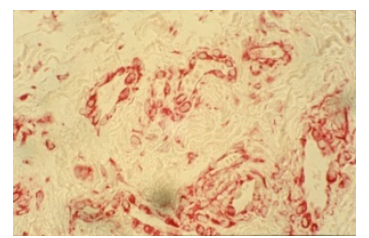

Figure I: Anti Factor VII staining with evidence of retrodiscal venous plexus; we can see many blood vessels with different dimensions, width and shape (I00X).

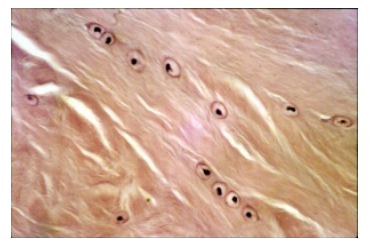

Figure 2: Hematoxylin- Eosin staining, that give evidence to condroid cells of the fibrocartilge of TMJ meniscus (I00X).

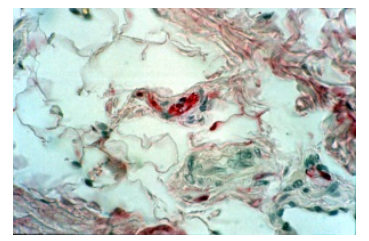

Figure 3: Anti S-100 Protein staining that give evidence to the presence of nervous fibers, and to a little neural receptor in fibro-vascular-adipous tissue (I60X).

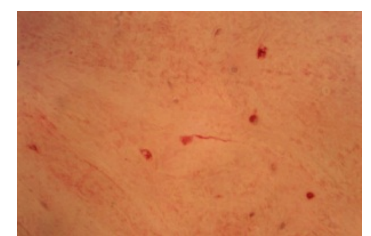

Figure 4: Anti S-100 Protein staining: we can see, near meniscus, a neural cell (I60X).

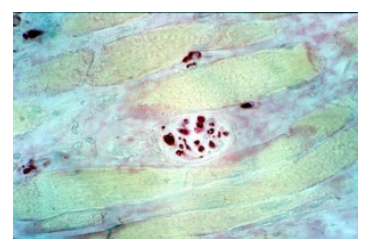

Figure 5: Anti MBP staining: there are some fibers of lateral pterigoid muscle, within some neural fibers (I00X).

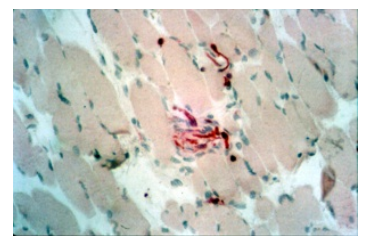

Figure 6: Anti MBP staining that give evidence to some neural fibers among muscular fiber cells in transverse section (I00X).

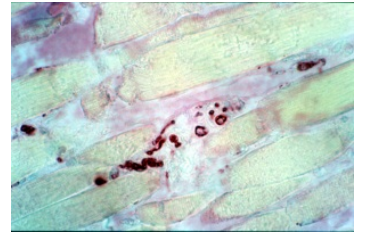

Figure 7: Anti MBP staining: we can see a particular of neural fibers among muscular fibers (I00X).

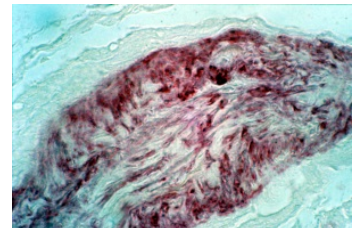

Figure 8: Anti MBP staining: we can see a Golgi-like receptor (I00X).

\section{Discussion}

Few studies are reported in literature on the precise identification and distribution of Nrec in articular and peri-articular tissues of TMJ. In the past, some authors (3-6) identified Ruffini's-like, Pacini's like and Golgi's like receptors both in articular and periarticular tissues, using conventional or histochemical methods usually performed to identify nerve fibres and receptors $(7,8)$. By using immunofluorescent techniques, other authors (3) demonstrated the presence of nervous fibers in the periarticular fibrous tissues, which seemed to run along the blood vessels reaching the fibrous cartilage of TMJ and ending to the inside.

Our study confirmed the results of other preceding reports on the presence of several different types of Nrec in periarticular soft tissues of TMJ, allowing additionally a precise immunohistochemical identification of Ruffini's-like, Pacini's- like and Golgi's-like receptors in skeletal muscles and tendons, in periartcular dense fibrous connective tissues and in subsynovial tissues. In fact, Nrec appeared NSE, S-100 and MBP immunoreactive showing GFAP and and Leu-7 immunoreactivity to lower degrees. Punctate reactivity for Neurofilaments and Synaptophysin was evident in the core of these receptors.

Free nervous endings, immunohistochemically positive for neurofilaments, NSE, and S100 protein, have been detected only in periarticular soft tissues ( higher density in muscles and in the vascular venous plexus in the posterior part of discal ligaments and in the trilaminar zone) and not in cartilagineous disk. The latter, besides, appeared constituted by S100 immunoreactive chondrocytes both in healthy individuals and in patients with chronic degenerative TMJ lesions. In pathological patients with severe disk damage number and morphology of chondrocytes were severely different in comparison to normal tis- 
sues (11); chondrocytes, in fact, were more numerous with a rough and thick elongated cytoplasmic processes conferring a dendritic-like appearance. In some instances, dendritic processes were extremely long and consequently their cytoplasm of origin could not be detected in a single section but only on consecutive sections of the same specimen. In no instances this cellular component and their prolongments in cartilagineous disks showed Neurofilaments, NSE or Synaptophysin immunoreactivity.

Morphological changes of chondrocytes in patients with chronic arthritic disease of TMJ we observed, conferring them the appearance of neural cells and their axons, have been previously described in literature (10). This misleading feature is further emphasized by the occurence of S-100 immunoreactivity in dendritic chondrocytes, as well as in normal chondrocytes. Nevertheless, S-100 positive cells in the articular cartilage of TMJ bear rare, thick and coarse cytoplasmic processes in which no one of the antigens commonly found in peripheral nerve fibres (e.g. Leu-7, MBP, Neurofilaments, NSE and Synaptophysin), except for S-100, could be detected. In contrast, peripheral nerve fibres usually exhibit long, thin and varicose cytoplamic prolongments in which a variable combination of the above mentioned antigens is usually detectable in conjunction with S-100 reactivity.

\section{Conclusions}

The results of our study indicate that free nervous endings described by other authors (3-8) are definitely proven to be Nrec, and it appears likely to stress that, especially in pathological conditions, chondrocytes and their prolongments might morphologically resemble Nrec in the articular disk, although their immunophenotype is rather different with the lack of expression of typical neural antigens.

Although immunochemistry can be easily used to study distribution and location of Nrec in articular tissues, we also suggest that ultrastuctural and immuno-ultrastructural studies should be performed in order to definitely assess if chondrocytes is the exclusive cell type of articular cartilage or if also Nrec could be present in TMJ.

\section{Competing Interests}

The authors have declared that no competing interest exists.

\section{References}

1. Rocabado M.: Arthrokinematics of the temporomandibular joint. Dent Clin North Am. 1983 Jul; 27 (3): 573-94.

2. Johansson AS, Isacsson G, Isberg A, Granholm AC. Distribution of substance P-like immunoreactive nerve fibers in temporomandibular joint soft tissues of monkey. Scand J Dent Res. 1986 Jun; 94 (3): 225-32.

3. Ichikawa H., Matsuo S,Wakisaka S., Akai M.: Peptidergic innervation of the temporomandibular disk in the rat. Experientia 1989 Mar 15;45(3): 303-4.
4. Klineberg I.J., Greenfield B.E., Wyke B.D.: Contributions to the reflex control of mastication from mechanoreceptors in the temporomandibular joint capsule. Dent Pract Dent Rec. 1970 Nov; 21(3):73-83.

5. Tahmasebi-Sarvestani A, Tedman RA, Goss A: Neural structures within the sheep temporomandibular joint. J Orofac Pain. 1996; 10(3):217-31.

6. Klineberg I.J., Greenfield B.E., Wyke B.D.: Afferent discharges from temporomandibular articular mechanoreceptors. An experimental analysis of their behavioural characteristics in the cat. Arch Oral Biol. 1971 Dec; 16(12): 1463-79.

7. Zimny M.L.: Mechanoreceptors in articular tissues. Am J Anat. 1988; 182: 16-32.

8. Wink C.S., Onge M.S., Zimny M.L.: Neural elements in the human temporomandibular articular disc. J Oral Maxillofac Surg. 1992 Apr; 50(4): 334-7.

9. Polak J, Van Noorden S. In: Immunocytochemistry: modern methods and applications. Bristol (UK): Wright PSG. 1986: 47-48

10. Bjornland T., Refsum S.B.: Histopathologic changes of the temporomandibular joint disk in patients with chronic arthritic disease. A comparison with internal derangement. Oral Surg Oral Med Oral Pathol. 1994 Jun; 77(6): 572-8.

11. Fujimura K., Kobayashi S., Suzuki T., Segami N: Histologic evaluation of tempromandibular arthritis induced by mild mechanical loading in rabbits. J Oral Pathol Med. 2005; 34: 157-163. 